Antoine G. Schneider

Marie-Hélène Perez

Piergiorgio Tozzi

Pierre Voirol

Patrick Schoettker

Anne Angelillo-Scherrer

Jacques Cotting

Ludwig Von Segesser

Philippe Eggimann

\section{Recombinant factor VIla for intractable life-threatening bleeding in patients with circulatory assist devices}

Accepted: 27 April 2010

Published online: 3 June 2010

(C) Copyright jointly held by Springer and ESICM 2010

Dear Editor,

Circulatory assist devices require tight anticoagulation therapy and may be complicated by severe bleeding. Despite warnings related to potential lethal thrombotic complications, offlabel use of recombinant factor VIIa ( $\mathrm{rFVIIa}$ ) is increasingly reported for refractory hemorrhage, including after cardiac surgery $[1,2]$. However, its use in patients with circulatory assist devices has seldom been reported and its safety remains to be established [3-5].

We reviewed 12 consecutive patients with surgically implanted devices who received rFVIIa as rescue treatment for intractable lifethreatening bleeding between March 2004 and November 2009 (Table 1). According to strict and constringent local consensus guidelines, a systematic cross-check for aggressive correction of hypothermia, acidosis, and coagulation factors with parallel surgical control for bleeding or embolization was obtained before rFVIIa administration in all cases.

The median age of patients was 45 years. The rFVIIa was administered in the operating room (3/12), in the intensive care unit (7/12), or both (2/12). Underlying conditions were cardiovascular surgery (11/12, including 4 heart transplantations) and bipulmonary transplantation (1/12). The device was an extracorporal membrane oxygenator (9/12) and a left ventricular assist device (3/12). Median dose of rFVIIa was $95 \mu \mathrm{g} / \mathrm{kg}(45-180)$, administered in one $(3 / 12)$ or several doses $(9 / 12)$.

In these otherwise terminal patients, the bleeding assessed by the number of $\mathrm{ml} / \mathrm{kg}$ of packed red blood cells and fresh frozen plasma requirement within $12 \mathrm{~h}$ before and after rFVIIa administration was significantly reduced. Six patients $(50 \%)$ died within 30 days, including 2 (18\%) from persistent bleeding. A careful analysis of coagulation and other physiological parameters did not reveal significant difference between responders and non-responders.

Table 1 Patient characteristics

\begin{tabular}{|c|c|c|c|c|c|c|c|c|c|c|c|c|c|}
\hline & \multirow[t]{2}{*}{$\begin{array}{l}\text { Age } \\
\text { (years) }\end{array}$} & \multirow[t]{2}{*}{$\begin{array}{l}\text { Type } \\
\text { of device }\end{array}$} & \multirow{2}{*}{$\begin{array}{l}\text { Total dose } \\
\text { of rFVIIa } \\
(\mu \mathrm{g} / \mathrm{kg})\end{array}$} & \multirow[t]{2}{*}{$\begin{array}{l}\text { Number } \\
\text { of doses }\end{array}$} & \multirow{2}{*}{$\begin{array}{l}\text { Thrombo- } \\
\text { embolic } \\
\text { event }\end{array}$} & \multirow[t]{2}{*}{$\begin{array}{l}\text { Device } \\
\text { clotting }\end{array}$} & \multicolumn{3}{|c|}{$\begin{array}{l}\text { PRBC requirements } \\
(\mathrm{ml} / \mathrm{kg})\end{array}$} & \multicolumn{3}{|c|}{$\begin{array}{l}\text { FFP requirements } \\
(\mathrm{ml} / \mathrm{kg})\end{array}$} & \multirow[t]{2}{*}{$\begin{array}{l}\text { Outcome at } \\
30 \text { days }\end{array}$} \\
\hline & & & & & & & $\begin{array}{l}12 \mathrm{~h} \\
\text { before }\end{array}$ & $\begin{array}{l}12 \mathrm{~h} \\
\text { after }\end{array}$ & $\begin{array}{l}\Delta \\
(\%)\end{array}$ & $\begin{array}{l}12 \mathrm{~h} \\
\text { before }\end{array}$ & $\begin{array}{l}12 \mathrm{~h} \\
\text { after }\end{array}$ & $\underset{(\%)}{\Delta}$ & \\
\hline 1 & 10 & ECMO & 180 & 2 & No & No & 96.0 & 75.0 & -22 & 26.8 & 35.7 & 33 & Alive \\
\hline 2 & $<1$ & ECMO & 180 & 2 & No & No & 270.0 & 77.0 & -71 & 160 & 42 & -74 & $\begin{array}{l}\text { Death day } 7 \text { from } \\
\text { multiple organ } \\
\text { failure }\end{array}$ \\
\hline 3 & 15 & ECMO & 45 & 1 & No & No & 22.8 & 35.9 & 57 & 10.9 & 21.7 & 99 & Alive \\
\hline 4 & $<1$ & ECMO & 180 & 2 & Stroke & No & 130.0 & 37.0 & -72 & 111 & 107 & -4 & $\begin{array}{l}\text { Death day } 2 \text { from } \\
\text { hemorrhage }\end{array}$ \\
\hline 5 & 55 & ECMO & 105 & 1 & No & No & 182.6 & 6.5 & -96 & 76.1 & 21.7 & -71 & Alive \\
\hline 6 & 51 & ECMO & 90 & 3 & No & No & 66.3 & 34.9 & -47 & 52.3 & 34.9 & -33 & $\begin{array}{l}\text { Death day } 9 \text { from } \\
\text { cardiogenic } \\
\text { shock }\end{array}$ \\
\hline 7 & 43 & LVAD & 100 & 2 & No & No & 105.0 & 40.0 & -62 & 75 & 20.8 & -72 & Alive \\
\hline 8 & 47 & ECMO & 54 & 1 & No & No & 31.4 & 45.3 & 44 & 34.9 & 49.4 & 42 & $\begin{array}{l}\text { Death day } 1 \text { from } \\
\text { hemorrhage }\end{array}$ \\
\hline 9 & 49 & LVAD & 60 & 2 & No & No & 33.8 & 11.3 & -67 & 25 & 6.3 & -75 & Alive \\
\hline 10 & 63 & LVAD & 76 & 3 & No & No & 34.7 & 6.3 & -82 & 34.2 & 5.3 & -85 & $\begin{array}{l}\text { Death day } 18 \text { from } \\
\text { septic shock }\end{array}$ \\
\hline 11 & 46 & ECMO & 126 & 2 & No & No & 88.4 & 18.9 & -79 & 63.2 & 26.3 & -58 & $\begin{array}{l}\text { Death day } 7 \text { from } \\
\text { septic shock }\end{array}$ \\
\hline 12 & 25 & ECMO & 47 & 2 & No & No & 144.0 & 56.0 & -61 & 100 & 43.3 & -57 & Alive \\
\hline
\end{tabular}

ECMO extracorporal membrane oxygenator; $L V A D$ Thoratec IVAD ${ }^{\circledR}$ as left ventricular assist device; $P R B C$ packed red blood cells $(300$ ml per unit), $\Delta p=0.007$ by Wilcoxon $T$ test; $F F P$ fresh frozen plasma $(250 \mathrm{ml}$ per unit), $\Delta p=0.007$ by Wilcoxon $T$ test 
In all cases, adequate anticoagulation was further maintained and no circuit thrombosis occurred; hence no device required replacement. One thromboembolic complication occurred (a stroke) in a neonate following cardiac arrest after a Jatene switch. The relationship between this event and the rFVIIa administration remained unclear.

We conclude that after meticulous control for all other potential contributing factors, the careful use of small and if necessary repeated doses of rFVIIa $(25-30 \mu \mathrm{g} / \mathrm{kg})$, allowed one to control life-threatening intractable bleeding in 9/12 patients with surgically implanted circulatory assistance devices without any clot of the devices. As suggested by current guidelines, these data confirm preliminary reports [3-5], but the safety of rFVIIa in this context should now be confirmed by controlled trials in larger groups of patients.

\section{References}

1. Karkouti K, Beattie WS, Arellano R, Aye T, Bussieres JS, Callum JL, Cheng D, Heinrich L, Kent B, Lee TW, MacAdams C, Mazer CD, Muirhead B, Rochon AG, Rubens FD, Sawchuk C, Wang S, Waters T, Wong BI, Yau TM (2008) Comprehensive Canadian review of the off-label use of recombinant activated factor VII in cardiac surgery. Circulation 118:331-338
2. Dunning J, Versteegh M, Fabbri A, Pavie A, Kolh P, Lockowandt U, Nashef SA (2008) Guideline on antiplatelet and anticoagulation management in cardiac surgery. Eur J Cardiothorac Surg 34:7392

3. Veldman A, Neuhaeuser C, Akintuerk H, Thul J, Gehron J, Schranz D, MichelBehnke I (2007) rFVIIa in the treatment of persistent hemorrhage in pediatric patients on ECMO following surgery for congenital heart disease. Paediatr Anaesth 17:1176-1181

4. Agarwal HS, Bennett JE, Churchwell $\mathrm{KB}$, Christian KG, Drinkwater DC Jr, He Y, Taylor MB (2007) Recombinant factor seven therapy for postoperative bleeding in neonatal and pediatric cardiac surgery. Ann Thorac Surg 84:161-168

5. Gandhi MJ, Pierce RA, Zhang L, Moon MR, Despotis GJ, Moazami N (2007) Use of activated recombinant factor VII for severe coagulopathy post ventricular assist device or orthotopic heart transplant. J Cardiothorac Surg 2:32

A. G. Schneider $(\bowtie) \cdot$ P. Eggimann

Division of Adult Intensive Care Medicine, Centre Hospitalier Universitaire Vaudois

(CHUV), Lausanne, Switzerland

e-mail: antoine.schneider@chuv.ch

M.-H. Perez · J. Cotting

Division of Pediatric Intensive Medicine,

Centre Hospitalier Universitaire Vaudois

(CHUV), Lausanne, Switzerland

P. Tozzi · L. Von Segesser

Division of Cardiovascular Surgery, Centre Hospitalier Universitaire Vaudois (CHUV), Lausanne, Switzerland
P. Voirol

Division of Hospital Pharmacy, Centre Hospitalier Universitaire Vaudois (CHUV), Lausanne, Switzerland

\section{P. Schoettker}

Division of Anesthesiology,

Centre Hospitalier Universitaire Vaudois

(CHUV), Lausanne, Switzerland

A. Angelillo-Scherrer

Division and Central Laboratory of Hematology, Centre Hospitalier Universitaire Vaudois (CHUV), Lausanne, Switzerland

A. G. Schneider - M.-H. Perez - P. Tozzi • P. Voirol · P. Schoettker .

A. Angelillo-Scherrer · J. Cotting ·

L. Von Segesser · P. Eggimann

Faculty of Biology and Medicine,

University of Lausanne, Lausanne, Switzerland 